\title{
ERROR DEL SISTEMA: HIPERADAPTACIÓN Y FRACASO EN EL NEÓN DE SIEMPRE, DE DAVID FOSTER WALLACE
}

\author{
SYSTEM ERROR: HYPERADAPTATION AND FAILURE \\ IN DAVID FOSTER WALLACE'S GOOD OLD NEON
}

\section{David SÁNCHEZ USANOS}

Universidad Autónoma de Madrid

\begin{abstract}
Resumen: Este artículo supone una lectura atenta del relato de David Foster Wallace El neón de siempre que lo conecta con otras piezas del autor y con otros documentos culturales que propician una reflexión acerca de nuestro presente, de la postmodernidad y de las contradicciones que plantea. El posible vínculo entre éxito social y fracaso personal, las paradojas a las que pueden conducir la razón y el cálculo, la relación entre competitividad y bloqueo emocional o la mercantilización de la experiencia serán algunas de las cuestiones que abordaremos en nuestro análisis, así como también los recursos retóricos y literarios empleados por David Foster Wallace para representar y confrontar un diagnóstico del que él mismo participaba.

Palabras clave: David Foster Wallace, postmodernidad, fraude, hiperadaptación, fracaso, mercantilización de la experiencia, bloqueo emocional, manipulación, metaficción.

Abstract: This paper is a close reading of David Foster Wallace's Good old neon. We connect this short story with other Wallace's works and with other documents of culture in order to think about our present time, about postmodernity and its contradictions. We analyze the possible ties between social success and personal failure, the paradoxes of reason and calculus, the link between competitiveness and emotional blockage and the commodification of experience. In addition to that we emphasize the literary and rethoric devices employed by David Foster Wallace with the purpose of represent and confront a diagnosis that was also his own.
\end{abstract}

Key words: David Foster Wallace, postmodernity, fraud, hyperadaptation, failure, commodification of experience, emotional blockage, manipulation, metafiction. 
Aquellos que han cruzado con mirada firme al otro reino de la muerte nos recuerdan — si acaso- no como perdidas almas violentas, sino sólo como los hombres huecos los hombres rellenos

T. S. Eliot, Los hombres huecos

A veces la literatura puede leerse como un corte, como una incisión a través de la cual podemos interpretar el mundo, analizar el tiempo que nos ha tocado vivir y, en última instancia, a nosotros mismos. Esa manera de leer, que hay quien se consideraría autorizado a llamar «filosófica», ni es la única ni tiene por qué resultar categórica; tampoco equivale a considerar la literatura como una disciplina ancilar de la ontología, de la moral o de la política, ni conlleva juicios acerca de las presuntas intenciones del autor. Nos gustaría pensar que en las siguientes páginas hemos adoptado una estrategia de lectura que incite al análisis y a la toma de distancia respecto al presente sin que ello signifique bloquear otras posibles interpretaciones ni encasillar al autor o a los textos que aquí pondremos en juego bajo ninguna «explicación definitiva» ${ }^{1}$.

David Foster Wallace es uno de los escritores angloamericanos más celebrados de las últimas décadas. La publicación que le proporcionó más notoriedad fue la novela La broma infinita (1996), aunque en nuestra opinión alcance más brillantez en relatos cortos como el que nos ocupa o en su faceta ensayística, caso del discurso de graduación Esto es agua (2005), algunas piezas de Algo supuestamente divertido que no volveré a hacer (1997), Hablemos de langostas (2005) o las entrevistas compiladas en Conversaciones con David Foster Wallace (2012).

El neón de siempre apareció en noviembre de 2001 en la revista Conjunctions y después fue incluido en la recopilación de relatos del autor Extinción en el año 2004 y es considerado como una de sus piezas más logradas ${ }^{2}$. La historia se sitúa en el presente (finales del siglo XX-comienzos del XXI) y a lo que más se parece es a una nota de suicidio, la que escribe o recita un hombre estadounidense con una trayectoria aparentemente exitosa según los parámetros habituales empleados para medir la integración y el prestigio social (profesión, número de experiencias sexuales, nivel de ingresos, etcétera). El narrador responde al nombre de Neal y elige como interlocutor a un personaje llamado David Wallace, que pertenecía al curso inmediatamente anterior en el instituto y que, como decimos, es a quien parece dirigida esta particular carta. Se trata de un texto escrito «desde el otro lado», pues está narrado en presente y en primera persona por alguien que dice haber estampado su coche contra uno de los pilares de un puente sobre la autopista diez años atrás, a las 21:17 del 19 de agosto de 1991 (el relato apareció impreso en 2001). El narrador-víctima parece ser un treintañero que reniega de la manera en la que ha conducido su vida, siempre dominada por el deseo de impresionar a los demás en

\footnotetext{
${ }^{1}$ Este artículo se enmarca dentro del proyecto de investigación «Failure. Reversing the Genealogies of Unsuccess, 16th19th Centuries» (Horizonte 2020, Marie Skłodowska-Curie Grant Agreement No 823998).

${ }^{2}$ Véase Peters (2014).
} 
función de logros visibles y cuantificables, guiando su comportamiento por el cálculo y la manipulación. El título (Good old neon en inglés) ya encierra cierta paradoja, pues por una parte transmite la idea de algo en lo que se puede confiar — algo artificial, en este caso-, pero la primera línea del texto se puede interpretar como un desmentido respecto a ese crédito: «Toda la vida he sido un fraude» (My whole life I've been a fraud). Ya la propia noción de la luz artificial connota cierto engaño o, al menos, cierto simulacro, pues consiste en desmentir la noche y la oscuridad, en conseguir una iluminación como la del día cuando no es de día (o allí donde la luz del día no alcanza). En este caso, el neón está explícitamente asociado al «aura» (artificial) que rodea a quienes son oficialmente admirados por los demás debido a sus éxitos deportivos, a su popularidad o a sus hazañas sexuales; en esos mismos términos se refiere a sí mismo Neal cuando especula acerca de la imagen de él que tendrá ese compañero de instituto, David Wallace, cuando, al enterarse de su muerte, curiosee en el anuario de su promoción para recordar quién era:

\footnotetext{
Y resulta que David Wallace tiene un conjunto enorme y en absoluto organizable de pensamientos intensos, sentimientos, recuerdos e impresiones sobre el tipo de esta pequeña foto que iba un año por encima de él en la escuela rodeado todo el tiempo de lo que parecía ser casi un aura de neón [neon aura] de excelencia escolástica y académica, de popularidad y de éxito con las señoritas (Wallace, 2012a, p. 219).
}

La idea de «fraude» omnipresente en el relato puede vincularse también con la de ciertas atracciones de feria, como ésas consistentes en unos espejos que devuelven la imagen deformada de uno mismo o esas otras - personas, dispositivos o artilugios - que vaticinan el futuro. En efecto, el narrador, situado en un enclave privilegiado (más allá de la vida, más allá del imperio y de la fícción que suponen el espacio y el tiempo secuencial ${ }^{3}$ ) parece estar desvelándole a su destinatario, David Wallace, que las cosas no eran como parecían, que su imagen — la de Neal — no se correspondía con su verdadera experiencia, con su realidad efectiva (interior) y que a lo mejor ambos se sentían igual de fracasados, que él, David, se equivocaba cuando reflexionaba

[...] sobre lo impresionante y auténticamente cómodo en el mundo que el tipo siempre parecía, como una persona viva de verdad en lugar del perfil o fantasma de una persona titubeante y patéticamente tímida que David Wallace se consideraba por aquel tiempo. Todo un tipo atractivo y lanzado al éxito de quien en la mejor tradición humana David Wallace había imaginado por entonces que era feliz e irreflexivo y no estaba en absoluto atormentado por voces que le decían que algo funcionaba terriblemente mal en él mientras que funcionaba bien en todos los demás (Wallace, 2012, pp. 219-220).

El carácter fraudulento de las imágenes, la disparidad entre esencia y apariencia y el espejismo o la falsedad asociadas a ésta última tiene una larga tradición en la historia de la filosofía, comenzando por la «condena» de Platón a la información procedente de los sentidos y su postulado acerca del carácter fantasmático de lo real presente en el símil de la caverna o la idea de «lo falso» (pseūdos) como uno de los modos de decir y pensar lo que es por parte de Aristóteles. La imagen que Neal ofrece

\footnotetext{
${ }^{3}$ Véase la nota del propio David Foster Wallace a su relato (pp. 218-219) en la que se menciona explícitamente la cuestión del carácter ficticio del tiempo secuencial. Una de las referencias filosóficas que acuden inmediatamente a la mente del lector es la misma Crítica de la razón pura en la que Immanuel Kant plantea el espacio y el tiempo no como entidades o relaciones pertenecientes al mundo sino como aspectos del sujeto humano (universal): como los requisitos necesarios para la percepción y la experiencia humanas (cf. con la «Estética trascendental» dentro de la mencionada Crítica de la razón pura). Sobre la cuestión del tiempo y el fraude en El neón de siempre puede consultarse Taheri (2013).
} 
al mundo - y en última instancia a sí mismo - es, por tanto, una falsificación — acabamos de ver cómo el narrador alude al «fantasma de una persona» (ghost of a person)—, un fraude que no tiene que ver ni con la felicidad ni con la salud que presuntamente proyecta; de hecho, el relato mismo parece una constante refutación de ambas cuestiones. Así, si en Aristóteles la idea de felicidad iba unida a la práctica de la virtud, nuestro narrador se encarga de ir desmontando retrospectivamente cada rasgo o comportamiento virtuoso que se le ha atribuido en el pasado y desvelándolo como un ejercicio de fría observación, fingimiento y cálculo; y si en el pensamiento clásico la salud estaba vinculada con la idea de equilibrio de humores y «ausencia de ruido», Neal es alguien que «estaba atormentado por voces que le decían que algo funcionaba terriblemente mal en él» (wholly unhaunted by voices telling him that there was something deeply wrong with him).

La idea de que el cuento se puede parecer a un ejercicio de profecía auto-cumplida, a una suerte de presencia espectral o voz de ultratumba que busca su satisfacción, se asienta no sólo en el hecho de que haya un personaje llamado como el autor (David Wallace, que también oye esas voces) sino también en lo que sabemos del David Foster Wallace real, que acabó suicidándose siete años después de la publicación de este relato en Claremont, California. (En este sentido nos parece magistral el título que puso D. T. Max a su biografía de David Foster Wallace: Todas las historias de amor son historias de fantasmas $^{4}$ ).

Podría conjeturarse, en la misma clave que asocia el fraude a lo especular y a lo fantasmal, que El neón de siempre es un mensaje que el propio David Foster Wallace se lanza a sí mismo, a su yo pretérito, a ese estudiante retraído que quizá miró con envidia — y con deseo de emulación — a algún «héroe» de instituto de los cursos superiores especialmente hábil con el deporte, con las chicas o con ambas cosas; y que cuando aquel chico tímido e inseguro alcanzó la fama vio que tampoco allí estaba el secreto que acallase las voces del malestar, que la vida, como aquel título de Kundera, que la vida estaba en otra parte y entonces, desafiando las leyes del espacio y del tiempo, trata de ponerse en contacto con su yo adolescente para trasmitirle este mensaje por persona interpuesta. En las jornadas que compartió en el año 1996 con el periodista de la Rolling Stone David Lipsky, Foster Wallace parece tener la misma voz auto-consciente (y auto-crítica) que el narrador de El neón de siempre a propósito de los valores más consolidados dentro de la sociedad de consumo, en este caso el atractivo (sexual) que puede conferir la fama:

Sólo a los Rolling Stones no les preocuparía eso. Y como soy consciente de ello, la cosa acabará en plan relajado. Pero, a ver, tiene que haber algo, porque está claro que las presentaciones se llenan y la gente se pone como a contonearse mientras leo o lo que sea. Pero según parece lo que yo quiero es no dar ningún paso. No quiero tener que decir «¿Te apetece venir al hotel?». Yo quiero que me digan «Te acompaño. ¿En qué hotel estás?» Y nadie hace eso (Wallace en Lipsky, 2017, p. 56).

El David Foster Wallace que responde a las preguntas del periodista de Rolling Stone se encuentra ante las mismas encrucijadas que el narrador de El neón de siempre: se ha educado dentro de los clichés de la sociedad de consumo, pero no puede —o no quiere - renunciar a ellos, quiere

\footnotetext{
${ }^{4}$ Además de la biografía de Max (2013) puede consultarse un texto muy crítico con la muerte y con la figura de Foster Wallace: el de Ziegler (2008).
} 
experimentarlos y, en todo caso, trascenderlos, superarlos desde dentro, atravesarlos, una estrategia que, en su caso, parece conducir al fracaso pues conlleva irremediablemente insatisfacción o culpabilidad, cuando no ambas cosas:

\footnotetext{
¿Pensabas que era factible?

No, pero tenía fantasías. Tenía una serie de fantasías sobre... Es muy raro, porque la mayoría de los aspectos de la fama no me importan. Pero de verdad pensaba, «A lo mejor mojo en esta gira». Ya, claro. Eso sería traicionar mi imagen y tienes razón. Visto en retrospectiva, ha sido una suerte que no lo lograse. Básicamente, al final me habría hecho sentir más solo. Porque no habría tenido nada que ver conmigo, sólo habría sido... (Wallace en Lipsky, 2017, p. 56).
}

En El neón de siempre el fracaso que se constata, el fraude vital que se anuncia desde sus primeras líneas tiene que ver con el cálculo y con la simulación / actuación: «Toda la vida he sido un fraude. No estoy exagerando. Casi todo lo que he hecho todo el tiempo es intentar crear cierta imagen de mí mismo en los demás. La mayor parte del tiempo para caer bien o para que me admiraran» (Wallace, 2012, p. 173). Desde el comienzo se plantea una oposición entre vivir y actuar, entre sentir de un modo genuino y atender a la construcción de un personaje, a la proyección de una imagen. En su fantástico ensayo de 1990, E unibus pluram: televisión y narrativa americana reflexiona sobre la influencia de la televisión en la configuración de la experiencia contemporánea y cómo ésta es abordada literariamente; el primer apartado de esas consideraciones lleva por título una desconcertante fórmula que pronuncian los regidores en los platós de televisión: «actúen con naturalidad». Foster Wallace, como buen lector de Wittgenstein, fue también un amante de las paradojas, más bien del carácter inagotablemente paradójico que se esconde casi tras cada giro del lenguaje, pero, en este caso, apunta a una cuestión aún más existencial: ¿cómo es posible actuar «con naturalidad»?, ¿cómo diferenciar la vida de un plató, el «como si» de la experiencia genuina? Foster Wallace vincula este carácter vacilante de lo real con la manera de ser propia de los escritores, con un modo de mirar que les confiere una forma radical de extranjería:

Los narradores como especie suelen ser mirones. Suelen acechar y observar. Son observadores natos. Son espectadores. Son esos tipos del metro cuya forma disimulada de mirar resulta inquietante. Casi depredadora. Es porque las situaciones humanas son el alimento de los escritores. Los narradores miran a otros seres humanos de la misma forma que los curiosos frenan para ver un accidente de coche: codician la imagen de sí mismos como testigos. Pero al mismo tiempo los narradores tienden a ser terriblemente conscientes de sí mismos. A la vez que dedican montones de tiempo productivo a estudiar con atención qué impresión produce en ellos la gente, los narradores también dedican montones de tiempo menos productivo preguntándose, nerviosos, qué impresión causan ellos a los demás. Qué tal caen, qué imagen tienen, si se les ve el faldón de la camisa por la bragueta, si tal vez tienen pintalabios en los dientes, si la gente a la que están mirando con disimulo los estarán considerando seres siniestros, como esos locos que acechan a la gente (Wallace, 2012b, p. 33).

La descripción que, en tanto que escritor, hace de sí mismo David Foster Wallace coincide casi con la de alguien que deambula entre los vivos pero que no pertenece a ellos, alguien que busca algo de los demás, no sólo como un voyeur, sino como un vampiro (forma de mirar «depredadora», pintalabios en los dientes, que acecha a la gente...), un tipo que habita ya entre los muertos y para el que los demás son su alimento, o su territorio en disputa, alguien con una relación muy torturada con la imagen y los espejos 


\begin{abstract}
Porque la razón de ser misma de la televisión es reflejar lo que la gente quiere ver. Es un espejo. No el espejo stendhaliano que refleja el cielo azul y el charco de barro. Más bien el espejo iluminado del baño ante el cual el adolescente calibra sus bíceps y decide cuál es su mejor perfil. Esta clase de ventana a la autopercepción nerviosa de los americanos tiene un valor incalculable a la hora de escribir narrativa (Wallace, 2012b, p. 34).
\end{abstract}

La voz del Foster Wallace de las entrevistas y ensayos se parece demasiado a la voz de El neón de siempre, alguien que no puede parar de observar, de medir y de medirse, que interpreta cualquier situación desde los parámetros de la compraventa o de la competitividad, incluso la misma terapia psicoanalítica a la que recurre el personaje, como casi todos los de su edad y condición que no logran ser felices a pesar de tenerlo aparentemente todo. Tampoco ese ámbito clínico escapa a la mirada disolvente del cálculo y de la imagen: la terapia es vista como un signo de estatus y una manera de proveerse de cierto vocabulario «de clase» (Wallace, 2012a, p. 174) y también como un contexto agonal en el que imponerse dialéctica e intelectualmente a su psicoanalista:

\footnotetext{
Y aunque le seguí el juego durante un rato a fin de no reventarle el globo, por dentro tenía una sensación bastante siniestra, porque ahora sabía que iba a ser igual de maleable y crédulo que el resto de la gente, no parecía tener nada ni siquiera cercano a la potencia de fuego que yo necesitaba para tener alguna esperanza de que alguien me ayudara a salir de la trampa de fraudulencia e infelicidad que me había construido a mí mismo (Wallace, 2012a, p. 188).
}

La insatisfacción permanente que exhibe el protagonista de El neón de siempre es un proceso que se inicia con el abandono de la infancia y que culmina con su suicido, un proceso que no parece aventurado calificar como «fracaso», algo sustentado, por lo demás, por el propio juicio que el narrador hace de sí mismo desde el comienzo, ofreciéndonos, a modo de tarjeta de visita, la antedicha fórmula: «toda la vida he sido un fraude». Según nuestro modo de verlo, este fracaso puede interpretarse como un caso de «hiperadaptación» al actual estado de cosas, a un presente que podríamos pensar a partir de nociones como las de «capitalismo tardío» (Ernest Mandel), «sociedad postindustrial» (Daniel Bell) o «postmodernidad»y «globalización» tal como las plantea Fredric Jameson. No obstante, lo esencial es atender a cómo el fracaso existencial / vital del narrador sobreviene precisamente por haberse ajustado en exceso a este presente, por haber seguido de manera estricta las normas y valores promovidos por esta sociedad de consumo, hija tal vez no tan ilegítima de la Ilustración.

En primer lugar, nos llama la atención el uso del término «fraude», una palabra con inequívocas connotaciones económicas y ligada en todo caso al engaño y a la proyección de una imagen falsa. El uso de categorías económicas en la obra de David Foster Wallace ha sido abordado por Jeffrey Severs en su David Foster Wallacce's balancing books: fictions of value (Severs, 2017); en general, recurrir a un vocabulario económico para abordar una crítica del presente - de aspectos del presente que no tienen que ver directamente con lo económico, se entiende - tiene un antecedente muy significativo en el uso que hace Lenin de «bancarrota» para describir la situación en que queda la Segunda Internacional en El Estado y la revolución (o en la obra que lleva por título La bancarrota de la II Internacional) o cuando habla de «la bancarrota moral de Herzen» en En memoria de Herzen ${ }^{5}$. Desde

\footnotetext{
${ }^{5}$ Véase Zea (1988), p. 78 y Piglia (2015), p. 170.
} 
coordenadas análogas podríamos decir que el «balance emocional» del protagonista de El neón de siempre arroja un saldo negativo debido al cálculo y al miedo: todo aquello que tiene que ver con lo sensorial, con la inmersión en una situación presente, con algo parecido a la plenitud parece bloqueado y sustituido por una conciencia vigilante siempre pendiente de la crónica pública de sus logros, de la imagen proyectada hacia el exterior.

\footnotetext{
El miedo me hacía esforzarme muchísimo, así que todo me iba siempre bien y terminaba consiguiendo lo que quería. Pero en realidad, en cuanto conseguía la mejor nota o ganaba el título deportivo de la ciudad o conseguía que Angela Mead me dejara ponerle la mano en el pecho, no sentía apenas nada más que tal vez el miedo a no ser capaz de conseguirlo otra vez. Recuerdo estar en la sala de recreo en el sótano de Angela Mead en el sofá y que ella me dejara meterle la mano por debajo de la blusa y no ser capaz de sentir la suavidad viva o lo que fuera de su pecho porque lo único a lo que yo me dedicaba era a pensar: «Ahora soy el tipo al que Angela Mead le ha dejado tocarle las tetas» (Wallace, 2012a, p. 173).
}

En esta pieza Foster Wallace, como tantos otros, vincula la vida adulta con la hipocresía, con el análisis, con la razón orientada a fines relacionados con la ganancia y la competitividad. Pareciera que la vida «auténtica» estuviese adscrita preferentemente a las sensaciones físicas, a los momentos de zambullida en lo circundante en los que calla la voz interior y no se piensa sino que se siente. Un modo de considerar las cosas que tiene numerosos antecedentes en nuestra cultura, desde ciertas posiciones de Nietzsche a las vanguardias artísticas y que encuentra en algunos de los versos del heterónimo de Fernando Pessoa, Alberto Caerio, una expresión muy lograda:

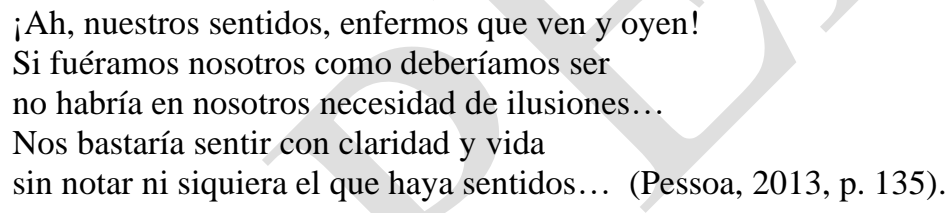

Así, en cierto momento, el protagonista de nuestro relato evoca su infancia y habla del «amor genuino» por el béisbol (genuine lovingly ball) que describe a propósito del olor de la hierba, del tacto de su equipación, de los sonidos del campo, del «clank» de los focos al encenderse

Y por encima de todo la sensación de salir al plato con la sensación de que cualquier cosa era posible, una sensación como si el sol estuviera resplandeciendo en algún lugar en lo alto de mi pecho. Y le conté [al psicoanalista] cómo ya a los catorce años todo aquello ya había desaparecido y se había convertido en preocupación por mis porcentajes individuales y por si podría ganar el torneo de la ciudad de nuevo (Wallace, 2012a, p. 191).

El momento de la anagnórisis, del reconocimiento trágico, parece llegarle a nuestro protagonista tras la muerte, desde esa atalaya de eternidad más allá del lenguaje y del tiempo desde la que habla, pues, de haberlo sabido antes, seguramente no hubiese recorrido el camino «oficial», aquel que parece dictarnos el statu quo en función del cual la promesa de la felicidad se encuentra en la acumulación de experiencias, en el consumo de vivencias, acontecimientos y logros, una operación que Neal lleva a cabo de manera diligente y exhaustiva y de la que nos ofrece una reveladora lista:

Estas son algunas de las cosas que probé: el Seminario Erdhard, conducir un vehículo de diez marchas hasta Nueva Escocia y de vuelta, la hipnosis, la cocaína, la quiropraxia sacro-cervical, unirme a una iglesia carismática, hacer footing, trabajar como voluntario para la oficina publicitaria del ayuntamiento, clases de 
meditación, los masones, el Forum Landmark, el Course in Miracles, un taller de dibujo en el hemisferio derecho del cerebro, el celibato, coleccionar y restaurar Corvettes antiguos e intentar dormir con una chica distinta todas las noches durante dos meses seguidos (acumulé un total de treinta y seis en sesenta y una noches y además pillé clamidia, hecho que les mencioné a mis amigos, fingiendo que estaba avergonzado pero confiando en secreto que la mayoría de ellos se quedaran impresionados - y pese a que se escudaron detrás de hacer bromas a mi costa, creo que lo estuvieron-, pero en su mayor parte aquellos dos meses únicamente me hicieron sentirme vacío y depredador, además dormí poquísimo y en el trabajo estaba hecho un asco; aquella fue también la época en la que probé la cocaína). Sé que esta parte es aburrida y que probablemente le esté aburriendo, pero se pone más interesante cuando llego a la parte en la que me mato y descubro lo que pasa inmediatamente después de que una persona se muere. En lo relativo a la lista, el psicoanálisis vino a ser lo último que probé (Wallace, 2012a, p. 175).

Hay pocas cosas más contemporáneas que «hacer listas», organizar la experiencia en función de series de cosas; en este caso particular, que casi podemos interpretar como paródico, se ve con especial intensidad el efecto de homologación que posee ese gesto: asistir a un seminario, a sesiones de quiropraxia o hacer voluntariado están al mismo nivel que probar la meditación, la cocaína, arreglar coches o mantener contacto íntimo con más de treinta chicas en un par de meses. La enumeración constata el carácter uniforme e intercambiable de los elementos de la lista, todo es considerado desde la perspectiva del consumo. Resulta muy significativa la constatación de que todo aquello le hizo sentirse vacío y «depredador» (predatory), un adjetivo ya empleado por el propio Foster Wallace cuando, en el texto sobre la influencia de la televisión en la narrativa contemporánea, describe el modo con el que los escritores se relacionan con sus congéneres, como vimos más arriba («Son esos tipos del metro cuya forma disimulada de mirar resulta inquietante. Casi depredadora [predatory]» (Wallace, 2012b, p. 33)). El narrador de El neón de siempre habla desde el centro de la sociedad de consumo que, como ya advirtiera William Burroughs, encuentra su epítome en la droga y su lenguaje; en ese sentido resulta paradigmática la presencia de la cocaína en esa lista, un término que aparece dos veces (al comienzo y al final de la enumeración), como signo de la verborrea y el olvido que en ocasiones van a asociados a su consumo. La lista se cierra con el psicoanálisis, una institución lingüística de carácter terapéutico que, por lo demás, es la que permite la estructura «confesional» del relato. Después de «probarlo todo», lo último que queda es esa conversación organizada con fines curativos presidida por el lenguaje y por la muerte (además el narrador se suicida y el psicoanalista fallece fruto de un cáncer), por la experiencia del tiempo y por la búsqueda de sentido, por los límites, en fin, que configuran la existencia humana, límites que el monólogo del narrador afirma haber trascendido con su fatal movimiento:

El doctor Gustafson y yo nos reímos bastante con esto después de que los dos muriéramos y nos situáramos fuera del tiempo lineal y en un proceso de cambio dramático, puede estar seguro. («El tiempo exterior» no es una simple expresión o forma de hablar, por cierto.) (Wallace, 2012a, p. 199).

La voz que nos habla en El neón de siempre nos transmite la idea — tan kafkiana ${ }^{6}$, por lo demásde que el mundo es una «trampa» (Wallace, 2012a, p. 206), un término (trap) que aparece mencionado explícitamente en el relato a propósito de la reposición de un capítulo de la serie Cheers en el que el nuestro protagonista se ve reflejado, concretamente cuando Lilith (que en la serie interpreta el papel

\footnotetext{
${ }^{6}$ A propósito de David Foster Wallace y Kafka la referencia inevitable es Wallace (2014).
} 
de una psicoanalista) comentando su día de trabajo dice: «Si me viene un solo yuppie más y empieza a lloriquearme sobre el hecho de que es incapaz de amar, voy a vomitar» (Wallace, 2012a, p. 205). La situación de reconocimiento no puede ser más postmoderna: viendo la reposición de una serie televisión; es ahí, en ese contexto metafictivo ${ }^{7}$, donde el protagonista ve quebrada de un modo un tanto siniestro su situación solipsista: no sólo descubriendo que no es único ni especial, sino que representa un cliché que sirve como material para el guión de una comedia de situación con risas enlatadas.

El carácter asfixiante de la razón y del lenguaje y la cuestión del solipsismo son preocupaciones nucleares para Foster Wallace, elementos que impulsan su narrativa y que tematiza en diversas ocasiones:

Heidegger es el tipo que la mayoría de la gente cree que nos metió en este bucle, pero cuando estaba trabajando en La escoba del sistema advertí que Wittgenstein era el verdadero arquitecto de la trampa posmoderna. Murió justo en el momento en que trataba la realidad como un hecho lingüístico en lugar de ontológico. Esto eliminó el solipsismo, pero no el horror. Porque todavía seguimos atascados. [...] Soy en el lenguaje. Somos en él. Wittgenstein no es Heidegger, no es que el lenguaje este en nosotros, sino que nosotros estamos en él, inevitablemente, igual que estamos en el espacio-tiempo de Kant (Wallace en Burn, 2012, p. 77).

Es como si tanto el David Foster Wallace «real» como el protagonista de El neón de siempre hubiesen asumido plenamente ciertas consideraciones que realiza Wittgenstein en el Tractatus y tratasen de sobrevivir con ellas

En rigor, lo que el solipsismo entiende es plenamente correcto, solo que eso no se puede decir, sino que se muestra.

Que el mundo es $m i$ mundo se muestra en que los límites del lenguaje (del lenguaje que solo yo entiendo) significan los límites de mi mundo.

El mundo y la vida son una y la misma cosa.

Yo soy mi mundo. (El microcosmos.)

(Proposiciones 5.62, 5.621 y 5.63 de Wittgenstein, 2007, p. 112).

Ese mundo de El neón de siempre y del propio David Foster Wallace, un mundo cerrado sobre sí mismo y sobrecargado de ironía y cultura de consumo, responde a la perfección a las características del ser humano en el estado de naturaleza de las que hablaba Hobbes en su Leviathan (1651): una vida en la que siempre se está a la defensiva, atacando o esperando ser atacado, una vida, en fin, organizada

\footnotetext{
${ }^{7}$ Metaficción que resulta en este punto recursiva, pues el propio David Foster Wallace en su ensayo acerca de la influencia de la televisión en la narrativa norteamericana reflexiona sobre el fenómeno de la irreverencia y la metaficción presentes en la televisión (y concretamente en las series de televisión), así como sobre la perversa relación que se establece entre televisión y expresión (ficticia) de una presunta individualidad excepcional, algo que se ejemplifica a la perfección en el caso de los anuncios: «Los productos que se presentan con la intención de ayudarlos a ustedes a expresar su individualidad solamente se pueden permitir anunciarse en televisión porque los compra una cantidad enorme de gente» (Wallace, 2012b, p.50). En este sentido podemos interpretar ese momento en el que el protagonista de El neón de siempre se ve reflejado en la serie Cheers como la contemplación siniestra e inesperada de un anuncio de sí mismo -en el que el producto es él, en tanto que objeto susceptible de tipificación codificada y burla en el medio de comunicación de masas por antonomasiay, siguiendo con las referencias a lo predatorio, como una situación en la que se descubre víctima de esa mentalidad irónica y consumista que la televisión en general y la publicidad en particular emblematizan: «Los anuncios de televisión siempre se dirigen a individuos, nunca a grupos, un hecho que parece curioso a la luz del tamaño sin precedentes del público de la tele, hasta que uno oye a los vendedores expertos explicar que la gente siempre es más vulnerable, y por tanto asustadiza, y por tanto fácil de convencer, si se los aborda cuando están solos» (Wallace, 2012b, p. 71). Sobre la metaficción en Foster Wallace véase Pijnappel (2011).
} 
en función de la máxima: «desconfianza, competitividad y gloria». De esa ontología y de esa axiología hablaba el David Foster Wallace real en su célebre discurso Esto es agua, cuando avisaba de los peligros (frustración y potencialmente suicidio) que conlleva el considerar a los demás, a «la gente», como un estorbo que se interpone en la consecución de mis metas individuales, como rivales en una presunta carrera (llegar a tiempo a casa, avanzar en la cola del supermercado). Se trata de un texto conciso, de prosa ágil y lírica que resulta difícil no leer desde unas coordenadas cuasi-biográficas que lo vincularían con El neón de siempre y E Unibus Pluram. Esto es agua es un discurso de graduación que, de nuevo, parece dirigido a un hipotético David Foster Wallace que estuviese sentado entre las filas de estudiantes recién titulados, en el que habla de él y para él y en el que podemos reconocer los fantasmas que le persiguieron durante toda su vida: la misma sobre-intelectualización de la que está aquejado el protagonista de El neón de siempre, la misma reluctancia a recibir ayuda o consejos, el mismo bloqueo ante lo sensorial, la misma huida respecto al presente:

Probablemente lo más peligroso que tiene la educación académica, por lo menos en mi caso, es que habilita mi tendencia a intelectualizar las cosas en exceso, a perderme en el pensamiento abstracto en lugar de limitarme a prestar atención a lo que está pasando delante de mí. Y en lugar de prestar atención a lo que está pasando dentro de mí (Wallace, 2012c, p. 3).

En lo que escribe David Foster Wallace hay siempre una lúcida cartografía del presente, de aquello en lo que consiste la vida cotidiana en la postmodernidad, una cotidianidad que, por cierto, en Esto es agua también está iluminada de manera artificial por los fluorescentes del supermercado, por la luz de la sociedad de masas y el capitalismo de consumo («una luz fluorescente y repulsiva (hideously, fluorescently lit)»), una realidad que nos programa para el egocentrismo y la competitividad, una realidad que normalmente es fuente de frustración y frente a la que a veces el suicidio parece la salida «lógica» (el único problema filosófico verdaderamente serio, el de si la vida merece la pena de ser vivida, como decía en 1942 Camus en El mito de Sísifo):

Todo esto no tiene nada que ver con la moralidad ni con la religión ni con las grandes y elaboradas preguntas sobre la vida después de la muerte. La verdad con V mayúscula tiene que ver con la vida antes de la muerte.

Tiene que ver con llegar a los treinta años, o incluso a los cincuenta, sin querer pegarte un tiro en la cabeza (Wallace, 2012c, pp. 4-5).

El suicidio aparece descrito en Esto es agua como un deseo de acallar esa voz incesante que no para de analizarlo todo y que no se muestra satisfecha con nada, un monólogo siniestro que parece ajustarse muy bien precisamente a la que caracteriza la narrativa de Foster Wallace,

No es para nada una coincidencia el que los adultos que se suicidan con armas de fuego casi siempre se peguen un tiro en... la cabeza.

Y la verdad es que la mayoría de esos suicidas en realidad ya están muertos mucho antes de apretar el gatillo (Wallace, 2012c, p. 4).

Una muerte en vida que nos recuerda inevitablemente al protagonista de El neón de siempre que, desde que abandonó la capacidad infantil de sumergirse en el presente y codificó la realidad en términos competitivos parece estar buscando la calma o la redención justamente con las estrategias 
diseñadas para todo lo contrario: para fomentar el consumo, querer siempre más y sentirse cada vez más solo. La hiperadaptación al sistema lleva aparejada la insatisfacción y la muerte, pues parece que la sociedad que hemos construido nos programa para que inevitablemente adoremos algo y ese algo, como si de un vampiro se tratase, termina por alimentarse de nosotros «Si adoras el dinero y las cosas materiales — si es de ellas de donde extraes el sentido verdadero de la vida—, entonces siempre querrás más. Siempre sentirás que quieres más» (Wallace, 2012c, p. 5); un esquema sacrificial que nos empuja a un sucedáneo de existencia, a un simulacro de vida que, cuando se la analiza con cierta distancia por quien la lleva, nos conduce a la noción sobre la que gira El neón de siempre y que también aparece en Esto es agua, el fraude: «Si adoras tu intelecto, el hecho de que te consideren listo, te acabarás sintiendo tonto y un fraude [ $\mathrm{a}$ fraud] y siempre estarás con miedo a que te descubran» (Wallace, 2012c, p. 5).

La salida de la espiral de autorreflexión, manipulación y narcisismo en la que se encuentra atrapado el protagonista de El neón de siempre sólo parece posible a través de la nota de suicidio, nota dirigida en cierto modo a David Wallace, como hemos visto, y en cierto modo a su hermana — primera víctima de sus ardides- , una nota en la que intenta poner en juego la sinceridad y el amor, haciendo examen de conciencia y pidiendo perdón. Es como si estos aspectos estuviesen penalizados o proscritos en el competitivo día a día de nuestro protagonista y sólo esa situación excepcional de poner fin a su vida le autorizase a hacerlo: «Resulta que hay cosas que se pueden tratar en una nota de suicidio pero que resultaría demasiado extraño expresar en otra clase de circunstancias» (Wallace, 2012a, p. 208). Resulta difícil no conectar este apunte con el lamento en clave generacional que el David Foster Wallace real expresa en diversos lugares acerca del desprestigio de la sinceridad, el amor o la convicción en el mundo moderno:

Me parece que la intelectualización y estetización de los principios y valores en este país es una de las cosas que ha destrozado a nuestra generación. Todo aquello que mis padres me decían, como «Es muy importante no mentir». Vale, controlado, lo tengo. Asiento pero en realidad no lo siento. Hasta que llego a los treinta y me doy cuenta de que, si te miento, yo tampoco puedo confiar en ti. Me siento dolido, estoy nervioso, estoy solo, y no puedo descubrir por qué. Y entonces me doy cuenta, «Ah, quizá el modo de abordarlo sea de verdad no mentir». La idea de que algo tan simple y, en realidad, tan estéticamente poco interesante - lo que para mí significaba que había que olvidarse de las cosas interesantes y complejaspueda ser verdaderamente sustancioso de un modo en que lo pícaro, lo meta, lo irónico y lo posmo no pueden me parece importante. Me parece que es algo que nuestra generación necesita experimentar (Wallace en Burn, 2012, p. 89).

Cuando el protagonista de El neón de siempre en la jornada en la que se va a suicidar emprende la escritura de su nota de suicidio también recupera de forma momentánea en el relato la concentración en los detalles sensoriales que echaba de menos cuando jugaba al béisbol por el placer del juego, sin la preocupación por batir marcas o impresionar a los demás: atiende al color de las hojas, a la nieve, a las sensaciones de lavarse los dientes o afeitarse, a las granjas cercanas a donde vive, a la puesta de sol, «Todo ello parecía temblar un poco, de esa forma en la que tiemblan las cosas que se reflejan en el agua» (Wallace, 2012a, p. 212). El carácter más genuino de la experiencia, que Foster Wallace termina vinculando siempre a lo sensorial y a lo infantil, reaparece casi de forma póstuma en El neón de siempre — de hecho ya hemos señalado que el relato tiene algo de mensaje de ultratumba-, como 
si constatase respecto a sí mismo el fracaso de la invitación que realiza a los (jóvenes) escritores del futuro al final de su texto sobre la influencia de la televisión:

Los próximos «rebeldes» literarios verdaderos de este país podrían muy bien surgir como una extraña banda de antirrebeldes, mirones natos que, de alguna forma, se atrevan a retirase de la mirada irónica, que realmente tengan el descaro infantil de promover y ejecutar principios carentes de dobles sentidos. Que traten de los viejos problemas y emociones pasados de moda de la vida americana con reverencia y convicción. Que se abstengan de la autoconsciencia y el tedio sofisticado. Por supuesto, esos antirrebeldes quedarían pasados de moda antes de empezar. Muertos en la página. Demasiado sinceros (Wallace, 2012b, pp. 99-100).

Neal nos ha estado contando su historia desde ese enclave más allá de la vida y del tiempo, nos ha estado hablando ya «muerto en la página» y ni siquiera rememorando lo que escribe en la nota de suicidio deja de ser ambivalente, tampoco entonces, en ese trance fatal, puede librarse de aquello que fue su condena: sigue pensando en el qué dirán a propósito de su nota, sigue intentando controlar qué efectos tendrán sus palabras en su hermana, en sus compañeros de trabajo, en los que le conocieron, cuánto hay de auténtico en su gesto y cuánto de teatral fruto de haberlo visto en tantas representaciones culturales previas, «Una parte de mí seguía calculando, actuando: y eso formaba parte de la naturaleza ceremonial de aquella última tarde» (Wallace, 2012a, p. 213). Podría contemplarse su suicidio (el de Neal pero también el de Foster Wallace) como una siniestra escapatoria a las paradojas en las que le encantaba regodearse ${ }^{8}$, como una forma de subrayar el carácter fatuo y opresivo de ciertos aspectos de la cultura (americana) de consumo. En efecto, como decíamos al comienzo de estas páginas, la literatura también puede leerse como un corte en el presemte que ilustra algunos de sus aspectos más característicos. Neal recurre a los fármacos (ingiere pastillas de Benadryl, que tienen efectos sedantes) antes de ponerse al volante de su coche para estrellarlo contra el pilar de uno de los puentes de la autopista, ¿qué hay más representativo de la realidad norteamericana que la industria farmacéutica y la automovilística? El empleo del coche como dispositivo para el suicidio tiene una larga tradición en dicha cultura: por muchos motivos el primer referente que acude a nosotros es el de Muerte de un viajante (1949), en el que el protagonista del drama, un viajante de comercio que lo ha fiado todo al éxito profesional, termina experimentando la soledad, la frustración y la locura (todo aquello que encaja bajo la consabida fórmula del «fracaso del sueño americano») y se suicida también con un coche, o el célebre final de la película Thelma \& Louise (1991) en la que, sintiéndose defraudadas por una vida muy alejada de sus sueños y sabiéndose acorraladas por la ley, la única salida que ven es saltar al vacío a bordo de un Ford Thunderbird, o aquellos versos de la canción Born to run (1975) de Bruce Springsteen en los que se describe a los coches como «máquinas suicidas»:

Por el día en las calles anhelamos

el fugitivo sueño americano.

Por la noche conducimos entre mansiones de gloria

en máquinas suicidas

que escapan de sus jaulas a la autopista 9.

\footnotetext{
8 «Es lo más cercano a cómo son las cosas de verdad. Es por eso por lo que son las paradojas lógicas lo que realmente vuelve chiflada a la gente. Un montón de grandes lógicos de la historia han acabado suicidándose, es un hecho» (Wallace, 2012a, p. 204).
} 
Ruedas cromadas, carburante inyectado y pisando más allá del límite.

Esta ciudad te arranca los huesos de la espalda.

Es una trampa mortal, es una invitación al suicidio.

Debemos salir mientras seamos jóvenes

porque vagabundos como nosotros, nacimos para correr. ${ }^{9}$

Otro ejemplo con dosis metafictivas sería el relato-homenaje que escribe Ray Bradbury a propósito del suicidio de Hemingway que lleva por título El invento Kilimanjaro (1965) en el que el coche se convierte en una singular máquina del tiempo en el que lector, autor y personaje se reúnen en una muerte legendaria, en un lugar mítico más allá del tiempo y del espacio, unas coordenadas, en fin, que parecen ser las mismas desde las que está narrado El neón de siempre. (También la poeta estadounidense Anne Sexton se suicidó con un coche, aunque en este caso asfixiándose con el monóxido de carbono procedente del tubo de escape.).

En definitiva, como sugeríamos, todo El neón de siempre puede leerse como una nota de suicidio en la que hasta su mismo final se reconocen los elementos que llevan a su protagonista a cometerlo - la cosificación y el anonimato propios del sistema al que se ha adaptado en exceso-, pues el texto concluye con una peculiar «firma», $\rightarrow \mathrm{NMN}$.80.418], que presuntamente alude a sus iniciales (NMN), el año de su promoción en el instituto (1980) y su promedio de bateo (.418); el narrador no finaliza con su nombre propio ni con un «te quiero» $\mathrm{u}$ otra fórmula que apuntase a esa sinceridad o a ese amor de que hablábamos antes, sino con un acrónimo y unas cifras, elementos que reproducen y consolidan la estandarización y la cuantificación de la vida que han tenido una presencia constante a lo largo de todo el relato. También nos parece significativo que la pieza termine hablando de (¿a?) David Wallace mientras éste se enfrenta a la voz de su conciencia - o de su alter ego-, el «exitoso» Neal que ha acabado suicidándose mientras tal vez él se consideraba un fracasado o un perdedor en el instituto en comparación con ese tipo del curso superior, una tensión que se zanja con la frase «Ni una palabra más (Not another word)» —estrictamente la última de El neón de siempre-, un precepto que casi coincide con la última entrada del diario de Cesare Pavese, otro conocido escritor suicida, que, en El oficio de vivir (1952), pocos días antes de arrojarse por la ventana, anota: «No palabras. Un gesto. No escribiré más» (Pavese, 2014, p. 403).

El suicidio del protagonista de El neón de siempre y tal vez el del propio David Foster Wallace podrían leerse, en fin, como una variante cultural actualizada y más siniestra del gesto de Sócrates -figura, como la de Foster Wallace, asociada a la ironía - de beberse la cicuta para subrayar a un tiempo la legitimidad pero también la injusticia de las leyes de la pólis, los destructivos parámetros en función de los cuales está planteada nuestra vida social, una trampa frente a la que no bastan la toma de conciencia, la reflexión o la crítica sino que tal vez haya que ir más allá —o más acá— del lenguaje.

\footnotetext{
${ }^{9}$ Adaptación de la traducción de Julià Salas, el original dice: «In the day we sweat it out on the streets / Of a runaway American dream / At night we ride through the mansions of glory / In suicide machines / Sprung from cages on Highway 9 / Chrome wheeled, fuel injected, and steppin' out over the line / Oh, baby this town rips the bones from your back / It's a death trap, it's a suicide rap / We gotta get out while we're young / 'Cause tramps like us, baby, we were born to run (Springsteen, 1975, p. 5).
} 


\section{Referencias bibliográficas}

BuRn, S. J. (ed.) (2012). Conversaciones con David Foster Wallace. Málaga, España: Editorial Pálido Fuego.

LIPSKY, D. (2017). Aunque por supuesto terminas siendo tú mismo. Un viaje con David Foster Wallace.

MAX, D. T. (2013). Every love story is a ghost story: a life of David Foster Wallace. Nueva York, Estados Unidos de América: Penguin.

PAVESE, C. (2014). El oficio de vivir. Barcelona, España: Seix Barral.

PessoA, F. (2013). Fernando Pessoa. Poesía I. Los poemas de Alberto Caeiro 1. Madrid, España: Abada.

Peters, T. (2014). Good old Wallace. Los Angeles Review of Books. Recuperado de https://lareviewofbooks.org/article/good-old-wallace/.

Piglia, R. (2015). Los diarios de Emilio Renzi. Años de formación. Barcelona, España: Anagrama.

PIJNAPPEL, J. (2011). «Not another word». Metafiction and solipsism in the short fiction of David Foster Wallace (BA Thesis English Language and Culture). Universiteit Utrecht.

SEVERS, J. (2017). David Foster Wallace's balancing books: fictions of value. Nueva York, Estados Unidos de América: Columbia University Press.

SpRINGSTEEN, B. (1975). Born to run. En Born to run [disco]. Nueva York, Estados Unidos de América: Columbia.

TAHERI, N (2013). Time and Fraud in David Foster Wallace's «Good old neon». Recuperado de: https://www.academia.edu/4969465/Time_and_Fraud_in_David_Foster_Wallaces_Good_Old_ Neon.

Wallace, D. F. (2012a). El neón de siempre. En D. F. WALlace, Extinción (pp. 173-221). Barcelona, España: Penguin Random House Grupo Editorial.

WALlaCE, D. F. (2012b). «E unibus pluram»: televisión y narrativa americana. En D. F. WALLACE, Algo supuestamente divertido que no volveré a hacer (pp. 33-100). Barcelona, España: Penguin Random House Grupo Editorial.

Wallace, D. F. (2012c). Esto es agua. Barcelona, España: Penguin Random House Grupo Editorial. Wallace, D. F. (2014). Algunos comentarios sobre lo gracioso que es Kafka, de los cuales probablemente no he quitado bastante. En D. F. WALLACE, Hablemos de langostas (pp. 79-84). Barcelona, España: Penguin Random House Grupo Editorial.

WitTGENSTEIN, L. (2007). Tractatus logico-philosophicus, Madrid, España: Alianza.

ZEA, L. (1988). Discurso desde la imaginación y la barbarie. Barcelona, España: Anthropos.

ZIEGLER, J. (2008). Death of a salesman. Editorial by John Ziegler. Recuperado de: http://johnziegler. com/editorials_details.asp?editorial=165. 